\title{
Secreted Wnt antagonists in leukemia: A road yet to be paved
}

\author{
Melek Pehlivan $^{\mathrm{a}, 1}$, Ceyda Çalışkan ${ }^{\mathrm{b}, 1}$, Zeynep Yüce ${ }^{\mathrm{c}}$, Hakki Ogun Sercan ${ }^{\mathrm{c}, *}$ \\ ${ }^{a}$ Vocational School of Health Services, Izmir Katip Celebi University, Izmir, Turkey \\ b Izmir Institute of Technology, Faculty of Science, Department of Molecular Biology \& Genetics, Izmir, Turkey \\ ${ }^{c}$ Dokuz Eylul University Faculty of Medicine, Department of Medical Biology and Genetics, Izmir, Turkey
}

\section{A R T I C L E I N F O}

\section{Keywords:}

Wnt signaling pathway

Secreted Wnt antagonists

CML

\begin{abstract}
A B S T R A C T
Wnt signaling has been a topic of research for many years for its diverse and fundamental functions in physiological (such as embryogenesis, organogenesis, proliferation, tissue repair and cellular differentiation) and pathological (carcinogenesis, congenital/genetic diseases, and tissue degeneration) processes. Wnt signaling pathway aberrations are associated with both solid tumors and hematological malignancies. Unregulated Wnt signaling observed in malignancies may be due to a wide spectrum of abnormalities, from mutations in the genes of key players to epigenetic modifications of Wnt antagonists. Of these, Wnt antagonists are gaining significant attention for their potential of being targets for treatment and inhibition of Wnt signaling. In this review, we discuss and summarize the significance of Wnt signaling antagonists in the pathogenesis and treatment of hematological malignancies.
\end{abstract}

\section{Introduction}

The first description of deregulated Wnt signaling in a hematological malignancy was reported in chronic myeloid leukemia (CML) [1]. CML is a clonal hematopoietic stem cell (HSC) disease characterized clinically by an increase in myeloid lineage cells at all stages of differentiation. The translocation $-\mathrm{t}(9 ; 22)(\mathrm{q} 34 ; \mathrm{q} 11)$ - leading to the formation of the Philadelphia chromosome (derivative 22) is the hallmark of CML. This translocation results in the fusion of the proto-oncogene ABL located on the long arm of chromosome 9, with the BCR gene on chromosome 22 [2]. The BCR-ABL oncoprotein possesses an unregulated increased tyrosine kinase activity that has been shown to drive the disease in terms of cell proliferation and resistance to programmed cell death [3]. There are several signaling pathways that have been determined to play a prominent role in CML progression. Among them the Wnt signaling pathway stands out with its unique role in the formation of a second leukemic stem cell population derived from granulocyte-macrophage progenitors. The neoplastic transformation of a HSC, results in the overproduction of granulocytes; thus forming the initial BCR-ABL positive leukemic stem cell (LSC) pool [4]. In the normal HSC, nuclear accumulation of $\beta$-catenin has been shown to be the driving force of self-renewal; its nuclear accumulation is therefore limited to HSCs in the bone marrow [5]. It is established that granulocyte-macrophage progenitors from advance stage CML patients display self-renewal capacity (a capability they normally do not possess), as a result of an overly active $\beta$-catenin/canonical Wnt signaling pathway, leading to two pools of $\mathrm{Ph}(+)$ cells with self-renewing capacity.

The importance of the Wnt signaling pathway in hematological malignancies is not limited to CML. Over-expression and aberrant regulation of Wnt signaling, mutations in downstream pathway members and silencing of Wnt antagonists by epigenetic regulation have all been reported in different hematological malignancies [6-8]. The epigenetic silencing of Wnt antagonists is of special interest and they are considered promising targets which carry the potential to be exploited in designing new agents and therapeutic strategies.

In this review, we focus on the role of Wnt antagonists in leukemia, with a special focus on CML; and discuss potential therapeutic opportunities presented by Wnt antagonists in hematological malignancies.

\subsection{The Wnt signaling pathway}

Wnt signaling is involved in many biological processes, including cell adhesion, migration, apoptosis, polarity, proliferation, development and organogenesis [9]. Secreted Wnt proteins act as ligands for the Frizzled (Fzd) receptor family and trigger paracrine/autocrine signaling through the Fzd proteins in the cell $[9,10]$. The ligand-receptor specificity between the 19 Wnt ligands and more than 15 receptors/coreceptors leads to the activation of intracellular Wnt signaling that is classified as canonical and non-canonical pathways; in which the

\footnotetext{
* Corresponding author at: Dokuz Eylul University Faculty of Medicine, Department of Medical Biology and Genetics, Inciralti, Izmir, 35340, Turkey.

E-mail addresses: melek.pehlivan@ikc.edu.tr (M. Pehlivan), ceyda.caliskan@ogr.deu.edu.tr (C. Çalışkan), zeynep.sercan@deu.edu.tr (Z. Yüce), ogun.sercan@deu.edu.tr (H.O. Sercan).

${ }^{1}$ These two authors have contributed equally.
} 
canonical pathway is responsible for preventing the degradation of $\beta$ catenin [11]. When Wnt signaling is not activated, $\beta$-catenin accumulation is prevented by its degradation by a multiprotein complex that is composed of Axin and adenomatous polyposis coli (APC) proteins (both defined as tumor suppressors), the Ser/Thr kinases GSK-3 and CK1, protein phosphatase $2 \mathrm{~A}$ (PP2A), and the E3-ubiquitin ligase $\beta$-TrCP. A $\beta$-TrCP recognition site is generated by this degradation complex through the phosphorylation of $\beta$-catenin on a conserved Ser/Thr-rich sequence near its amino terminus; thus marking it for degradation by the proteasome [12].

Canonical Wnt signaling is triggered by the binding of the Wnt ligand to the Fzd receptor in the presence of the co-receptor LRP5 or 6 (Low-density lipoprotein receptor-related protein) which in turn transfers the signal into the cell [5,9]. Receptor-ligand coupling results in the phosphorylation of Dishevelled (Dvl) proteins. The resulting Dvl/ Fzd receptor complex stimulates the formation of LRP aggregates in the cell membrane and $\mathrm{CK} 1 \gamma$ phosphorylation of the intracellular domains of the LRP receptors. These signaling events lead to Axin being trapped by the receptor complex. Phosphorylated Dvl disrupts the Axin-APCGSK3 $\beta$ degradation complex, inhibiting the phosphorylation of $\beta$-catenin and suppressing proteosomal degradation. As a result, $\beta$-catenin levels rise in the cytoplasm and translocate to the nucleus. Nuclear $\beta$ catenin interacts with members of the Tcf/Lef transcription factor family and binds to co-activator proteins converting them into transcriptional activators of Wnt target genes [13].

Non-canonical Wnt pathways are defined as pathways that do not involve $\beta$-catenin stabilization. Presently eleven different non-canonical Wnt pathway has been identified (eg Wnt/RAP1, Wnt/Ror2, Wnt/PKA, Wnt/GSK3, Wnt/RYK, Wnt/mTOR ect.). Some of these pathways also use/interact with Fzd transmembrane proteins as receptor. Non-canonical pathways in which Fzd receptors are involved are divided into Wnt/planar cell polarity (PCP) and Wnt/ $\mathrm{Ca}^{2+}$ pathways [14].

In Wnt $/ \mathrm{Ca}^{2+}$ signaling, intracellular calcium release is triggered by the binding of the Wnt ligand to its Fzd receptor, followed by G protein signaling leading to PKC-mediated cleavage of phosphatidolinoside to diacylglycerol and inositol 1,4,5 triphosphate. Activation of secondary messengers triggers intracellular calcium release resulting in calcium dependent kinase activation such as CamKII and CaCN. These kinases activate the expression of transcriptional co-activators such as NFAT and NFkB [15]. This pathway is known to play an important role in regulating cell adhesion, cell migration, embryonic development of dorso-ventral patterns and development of the heart [16].

The Wnt/PCP pathway plays important roles in regulating morphogenic polarization, cell fate, embryonic morphogenesis and cellular mobility [16]. It's activated by the binding of ligands that function in non-canonical signaling such as Wnt5a, Wnt5b, Wnt11, to Fzd family proteins or ROR2, ROR1 and Ryk receptors [17]. Similar to LRP5/6, ROR1 and ROR2 are phosphorylated by GSK3 $\beta$ and interact with Dsh proteins after Wnt5a binding. As a result a Fzd/ROR active receptor complex is formed [15]. This complex activates Dvl which in turn transduces the signal to small GTPases and JNK (C-Jun NH2-terminal kinase) via R protein, $\mathrm{G}$ protein, RhoA, Rac and Cdc42 [18].

Recent studies have identified a new "alternative Wnt pathway" in which $\beta$-catenin is replaced by YAP/TAZ proteins. YAP/TAZ proteins are transcriptional regulators of the hippo pathway, the key regulator of organ size and tissue homeostasis [19]. In the absence of Wnt ligands, $\mathrm{YAP} / \mathrm{TAZ}$ allows aggregation of $\beta$-TrCP and acts as a negative regulator of the Wnt pathway. When Wnt signaling is activated, YAP/TAZ and $\beta$ $\operatorname{TrCP}$ are removed from the degradation complex by Axin1. Free YAP/ TAZ on the contrary, acts as a positive transcriptional regulator of the Wnt pathway [20]. Park et al. have shown YAP/TAZ accumulates in the nucleus through Wnt5a/b and Wnt3a and transcriptionally regulates the expression of various genes [19].

Recent and ongoing studies are constantly identifying new molecules associated with Wnt signaling. Of these, newly identified Wnt agonists, Norrin and R-Spondin are worth mentioning [21]. Norrin binds directly to the Fzd4/LRP5 complex and activates signal transduction. R-Spondin binds to G-protein-coupled receptors (Lgr4-6) and inhibits the ubiquitination and degradation of Fzd proteins [22,23]. Both proteins are described as positive regulators of the Wnt pathway.

\subsection{Wnt signaling in Hematological Malignancies}

It is well established that the Wnt/ $\beta$-catenin pathway controls the proliferation, survival and differentiation of hematopoietic cells [24]. Continuous stimulation of Wnt signaling results in the neoplastic transformation of myeloid and lymphoid lineages. Physiologically the pathway is regulated stringently [25]. In healthy cells $\beta$-catenin levels are tightly controlled by secreted and/or intracellular located inhibitory proteins. Epigenetic abnormalities and silencing suppressors of the pathway trigger the increase of $\beta$-catenin levels, leading to the uncontrolled activation of the Wnt signaling pathway $[26,27]$.

Canonical Wnt signaling is significant for the maintenance and establishment of fetal HSCs [28]. Wnt signaling can directly promote HSC self-renewal and has the ability to reconstitute the hematopoietic system of lethally irradiated mice [29]. Wnt signal strength was shown to regulate normal hematopoiesis [30]. Research over the past years has led to our understanding that Wnt signaling is a critical regulator of distinct aspects of self-renewal and differentiation in stem cells of the hematopoietic system [28]. The differential expression of canonical Wnt signaling was shown to have opposing effects on HSCs. Constitutive activation of $\beta$-catenin has been demonstrated to induce HSC re-population ability and inhibited differentiation [31]. On the other hand non-canonical Wnt signaling also plays an important role in HSC physiology. Non-canonical Wnt signaling maintains quiescent longterm HSCs the bone marrow niche and has been shown to have a role in the aging of HSCs [32,33].

Being a central element of HSC development and maintenance, it is not surprising that the deregulation of this pathway plays a role in leukemia development and progression. Clinical and experimental studies have shown that Wnt signal transduction is impaired in hematological malignancies. The most apparent result of deregulated Wnt signaling is the increase of intracellular $\beta$-catenin levels. Sadras et al. have shown that $\beta$-catenin expression is upregulated in primary AML cells and is associated with enhanced clonogenic and self-renewal capacity [34]. The aberrant activation of Wnt/ $\beta$-catenin has also been implicated in the development and progression of acute lymphoblastic leukemia (ALL). The expression levels of Wnt ligands, effects dose dependent regulation of Wnt signaling in hematopoiesis [31]. Wnt ligands WNT2B, WNT5A, WNT10B, WNT16B and Wnt receptors FZD7 and FZD8 were reported to be overexpressed in B cell progenitor ALL cells and primary B-ALL cells. LEF1 is also shown to be overexpressed in ALL, CLL and malignant lymphoma [35].

Wnt signaling is known to prolong leukemic stem cell (LSC) survival. Similar to its effects on HSC's, Wnt/ $\beta$-catenin signaling is required for the self-renewal of LSCs [36]. Additionally canonical Wnt signaling has anti-apoptotic effects in the LSCs of leukemia models when challenged with anti-cancer drugs [6]. Wnt signaling especially has a pivotal role in the different compartments of CML-LSC development [37]. $\beta$-catenin overexpression is observed in the LSCs during the accelerated stage of disease and blast crisis [1]; pointing to the canonical Wnt pathway as a good therapeutic target in CML-LSCs.

Epigenetic dysregulation is another cause of the Wnt/ $\beta$-catenin pathway dysfunction in solid tumors and hematological malignancies [38]. Promotor methylation of Wnt antagonists were detected in multiple types of hematological malignancies and correlated with poor prognosis. The epigenetic silencing of one or multiple Wnt antagonists results in the constitutive activation of the pathway. The presence of chimeric oncogenes such as AML1/RUNX1, MLL/PTD, PML/RAR $\alpha$, has been reported to correlate with the hyper-methylation and silencing of Wnt inhibitors [35].

Although most research conducted on Wnt signaling in 
hematological malignancies focus on the Wnt/ $\beta$-catenin pathway, interest in non-canonical signaling is on the rise. Non-canonical pathways have been reported to function as tumor suppressors in mouse myeloid and lymphoid leukemia models [39]. Similar to the canonical Wnt pathway, non-canonical signaling is involved in the self-renewal and maintenance of HSCs. It has been shown that two player of non-canonical signalling -Flamingo (Fmi) and Fzd 8- maintain quiescent longterm HSCs [33]. Wnt5a signals through the non-canonical Wnt/Ca ${ }^{2+}$ pathway and has been shown to supress cyclin D1. Research on animal models suggests it acts as tumor suppressor. WNT5a expression was inhibited in ALL patients by promoter methylation, which correlated with upregulation of cyclin D1 expression and poor prognosis[40]. Another study has shown that targeting the non-canonical Wnt/ $\mathrm{Ca}^{2+}$ pathway leads to impaired NFAT activity and decreased cytokine production, increasing sensitivity to Bcr-Abl inhibition in a mouse model of Bcr-Abl + ALL [41].

Uncontrolled activation of the Wnt/PCP pathway leads to more malignant phenotypes, disruption of tissue polarity, invasion, metastases in human tumors [17]. Deregulated WNT/PCP signaling drives the pathogenesis of chronic lymphocytic leukemia (CLL). Targeting casein kinase (CK) $1 \delta / \varepsilon$, a key component of this pathway has synergetic effects on therapeutic strategies based on inhibiting B-cell receptor (BCR) signaling [42]. Furthermore The Wnt/PCP pathway co-receptor ROR1 was found to be expressed on the surface of CLL cells and not on mature healthy B cells; thus presenting itself as a target for future therapeutic strategies [43-45].

\subsection{Wnt Inhibitors/Antagonists}

Wnt antagonists are an additional Wnt signaling balancing mechanism, evolutionally gained in vertebrates. Wnt antagonists resemble guards controlling Wnt signaling pathway activity. So far six family members of intracellular and secreted Wnt signaling inhibitors: Dickkopf proteins (Dkks), secreted Frizzled-related proteins (sFRPs), Wnt-inhibitory factor 1 (WIF-1), Wise/SOST, Cerberus, insulin-like growth-factor binding protein 4 (IGFBP-4); and four family members of transmembrane proteins: Shisa, Wnt-activated inhibitory factor 1 (Waif1/5T4), adenomatosis polyposis coli down-regulated 1 (APCDD1), and Tiki1 proteins, have all been defined as acting as Wnt antagonists [46]. The common mode of action is to prevent binding of Wnt ligands to their cell surface receptors, thereby inhibiting Wnt/receptor stabilization and further pathway activation. Many studies have shown that the downregulated expression of Wnt antagonists via epigenetic inactivation plays a significant role in the etiopathogenesis of different forms of cancer. Abnormal changes in expression levels of these antagonists are reported to be related with decreased survival in several hematological malignancies [35]. Though we know that Wnt antagonists play a significant role in the regulation of Wnt signaling; research on the pathophysiological effects of their dysregulation in hematologic malignancies is limited. Some Wnt antagonists interact directly with Wnt proteins while others act on the Wnt receptor complex. Wnt antagonists also have different transcript isoforms and not all of them act like an antagonist, adding to the complexity of the regulation of Wnt signaling. Below are the most studied Wnt antagonists; Dickkopf, sFRP1, WIF1, SOST, WNT5a and their role in hematological malignancies (Fig. 1).

\subsubsection{Dickkopf (DKK)}

Dickkopf proteins are secreted extracellular antagonists of Wnt signaling. Four isoforms of the Dickkopf protein have been identified in vertebrates: Dkk1, 2, 3, 4 [47]. Dkk1, 2 and Dkk4 can block canonical Wnt signaling by inhibiting the function of the LRP5/6 coreceptors, but they do not function in non-canonical Wnt pathways such Wnt/PCP and Wnt $/ \mathrm{Ca}^{2+}$ signaling [48]. Dkk1 is the most well-known and studied protein isoform of Dkks. Dkk1 forms a complex with LRP5/6 and Kremen (Krm), resulting in the endocytosis of the complex and sequestration of LRP5/6 from the cell surface [49]. Recombinant Dkk1 protein has been used as an efficient Wnt/ $\beta$-catenin pathway inhibitor, in vitro settings [50]. In addition, Dkk1 was shown to inhibit proliferation and induce apoptosis in human renal cell carcinoma [51].

Dkks have an affinity for Krm proteins [52], by which they can induce the proteasomal degradation of $\beta$-catenin [53]. Interestingly Dkk2 can function as a Wnt/LRP6 signaling agonist or antagonist and both functions are mediated through Krm2. It must be noted that Dkk2 alone cannot activate the Wnt/ $\beta$-catenin signaling and that the LRP6 levels modulate Dkk2-mediated activation of the Wnt pathway [54]. The effects of DKK3 on Wnt signaling are controversial and may differ between tissue and tumor cell types. For example; DKK3 was reported to selectively activate the c-Jun-NH2-kinase in human prostate cancer cells, leading to the inhibition of the non-canonical Wnt pathway [48].

There are a couple of studies examining the role of the Wnt antagonist Dkk proteins in leukemia. Staal et al. showed that DKK3 is epigenetically downregulated by hyper methylation, resulting in an over active Wnt signaling in the primary CLL and B-ALL cells [8]. It was demonstrated that in addition to promoter hyper methylation, posttranslational mechanisms also regulates DKK3 expression in adult BALL [48]. The relative expression of DKK3 was lower in bone marrow cells of AML and ALL patients than in normal controls [55]. There is evidence that miR-708 regulated the expression levels of Dkk3 and in the presence of a miR-708 inhibitor Dkk3 expression levels increase, independent of promoter demethylation. Restoration of Dkk3 leads to the suppression of the Wnt/ $\beta$-catenin signaling pathway and consequently suppression of cell growth [48]. Hyper methylation of DKK1 has also been associated with the activation of the Wnt pathway and poor prognosis in AML patients [8]. Dkk1 is overexpressed in multiple myeloma (MM); thus termed as one of the pro-myeloma factors [56]. Tian et al. demonstrated that Dkk1 levels increase in MM patients [57] and the neutralization of Dkk1 may have beneficial effects in the treatment of MM [50]. Liang et al. reported that cordycepin -an adenosine analogue- induces Dkk1 expression via autocrine and paracrine pathways in leukemia and mesenchymal stromal/stem cells (MSCs). Paracrine Dkk1 secreted from MSCs was shown to inhibit leukemia and cancer cell proliferation [58].

Several studies aimed at determining whether exploiting Dkk-1 signaling may be effective in leukemia treatment. In one study the addition of recombinant Dkk1 protein to CLL cells failed to inhibit Wnt signaling [59]; implying that although actively downregulated, Dkk1 alone is not sufficient in suppressing Wnt signaling in chronic leukemia such as CML and CLL.

\subsubsection{Wnt Inhibitory Factor 1 (WIF1)}

WIF1 is a Wnt binding protein that prevents Wnt ligands to bind with their associated membrane Frizzled receptors. Structurally WIF1 contains a N-terminal secretion signal sequence, WIF domain (WD, 143 amino acid residues), five epidermal growth factor (EGF) -like domains (each 31-33 residues), and a hydrophilic C-terminal. The human Wif1 protein has been shown to be able to bind eight different secreted Wnt proteins through the WD: Wnt3a, Wnt4, Wnt5a, Wnt9a, Wnt11, Wingless and Xenopus Wnt8 [60]. Wif proteins inhibit Wnt/ $\beta$-catenin signaling by sequestering Wnt ligands [61]. Unlike Dkk proteins, Wif1 inhibits both canonical and non-canonical pathways [62].

$\mathrm{Wnt} / \beta$-catenin signaling is stringently regulated in normal tissues where Wif1 is one of the players that modulate Wnt/ $\beta$-catenin signaling activity. Functional loss of Wif1 by promoter hyper-methylation leads to the activation of Wnt signaling, resulting in uncontrolled and increased cell proliferation in a variety of cancers, such as colorectal, prostate, melanoma, lung, breast, esophagus, stomach and bladder [63]. Overexpression of WIF1 has been reported to inhibit cell growth in lung and bladder cancers in vitro; another example in which the down regulation of a Wnt pathway antagonist is observed during carcinogenesis and when restored has the ability to inhibit growth of cancer cells [60]. 


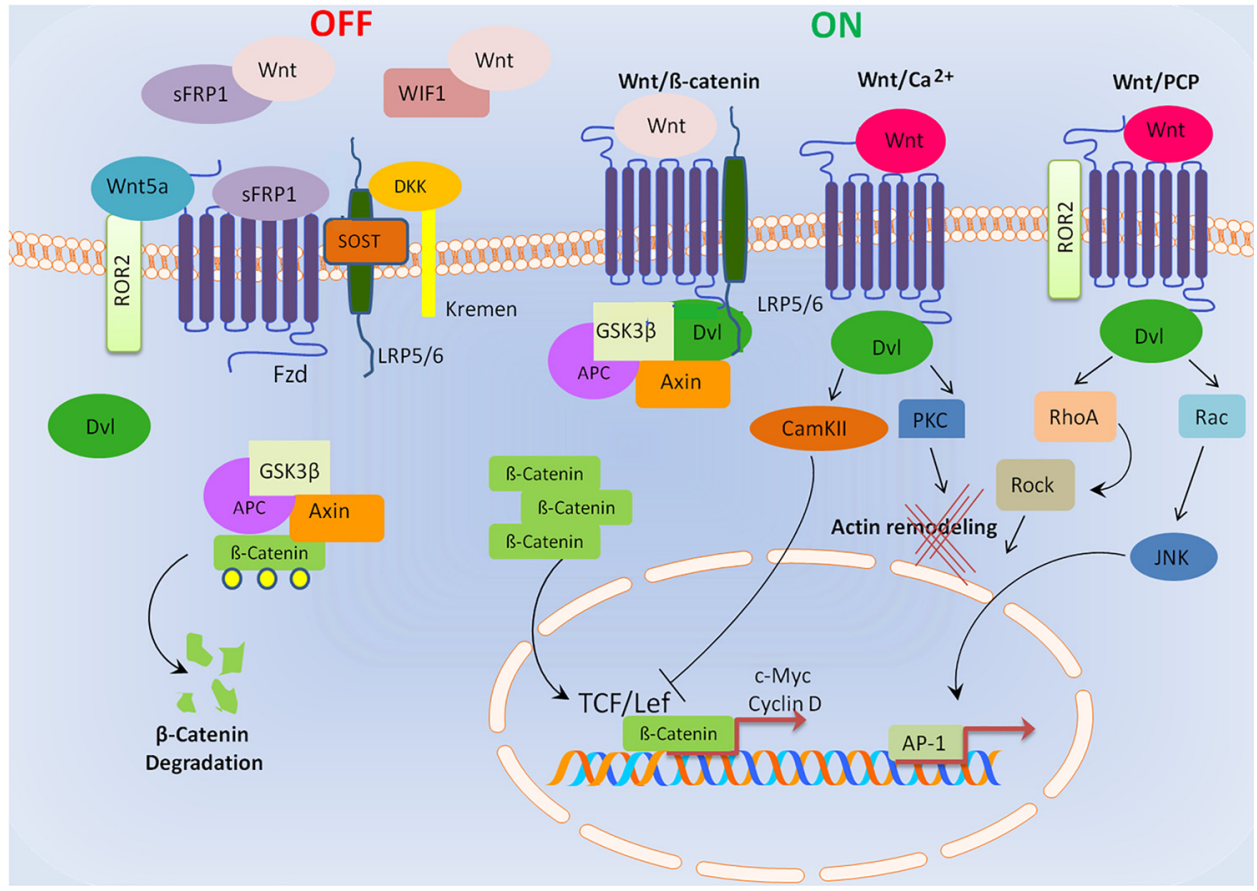

Fig. 1. Regulation of Wnt signalling by antagonists. Canonical Wnt/ $\beta$-catenin pathway: In the presence of Wnt antagonists (OFF state) Wnt/receptor interactions are interrupted and $\beta$-catenin is rapidly degraded via ubiquination at the proteosome by the formation of the destruction complex (APC, Axin, GSK3 $\beta$ ). In the absence Wnt antagonists ( $O N$ state) Wnts form a complex with the Frizzled receptor and coreceptors LRP5/LRP6, resulting in the inhibition of the destruction complex. This inhibition leads to $\beta$-catenin accumulation in the cytoplasm, followed by its translocation to the nucleus where it forms a complex with TCF/LEF transcription factors and initiates transcription of Wnt target genes. Non-canonical Wnt/Ca2+ pathway: Interaction of Wnt ligands with Fzd receptors can lead to a release of intracellular $\mathrm{Ca}^{2+}$. Intracellular calcium subsequently activates CAMKII and PKC in cells. Non-canonical Wnt/PCP pathway: Wnts bind to transmembrane receptors ROR2 to activate planar cell polarity. ROR2 activation leads to the activation of small GTPases, Rho and Rac; which in turn activates JNK. The signaling cascade results in activation-specific gene transcription through modulation of the AP-1 protein complex.
There is limited research on the role of Wif1 in leukomogenesis. The first report of Wif1 epigenetic down regulation in a hematological malignancy was observed in CLL patients. In this study Wif1 was shown to be a negative regulator of activated Wnt signaling in CLL [64]. In another study Ghasemi et al. reported that WIF1 and DKK1 hyper methylation were observed in AML patients; the observed hyper methylation was relatively much higher in undifferentiated phenotypic subgroups (AML M0) when compared to more differentiated ones (AML M5). This study was compared to others where lower rates of methylation were observed in AML patients; concluding that the observed differences may be due to different patient selection and their ethnic differences [65]. Regarding therapeutic interventions, Pehlivan et al. showed that induced WIF1 expression will sensitize CML cells to tyrosine kinase inhibitors, again making Wnt signaling a promising target in TKI resistant patients. The WIF1 gene was reported to be hypermethylated in CML cell lines [27]. However, to date there has been no reports on the expression levels and regulatory effect of WIF1 in CML patients.

\subsection{3. sFRP (Secreted Frizzled Related Protein)}

The sFRP family of Wnt antagonists is comprised of sFRP proteins numbered 1 through 5 [66]. sFRPs regulate Wnt signaling by binding to Wnt ligands through their CRD domains, similar to those found on FZD receptors. sFRPs can interfere with Wnt signaling by forming nonfunctional complexes with Fzds or by interacting with Wnt proteins to prevent them from binding to their Fzd receptors $[67,68]$.

The sFRP gene family is often the target of epigenetic silencing by DNA methylation. Changes in the expression of sFRP genes have been demonstrated in various cancers such as colorectal, bladder, prostate, endometrium, lung, breast, mesothelioma and chronic lymphocytic leukemia. These observations have led to the mutual understanding that sFRPs function as tumor suppressors [69]. Unlike Dkks, which specifically inhibit Wnt/ $\beta$-catenin signaling, sFRPs can inhibit non-canonical Wnt/PCP signaling, as they bind to both types of Wnts [46].

Being natural inhibitors of both canonical and non-canonical Wnt signaling, sFRPs play important roles in the differentiation of hematopoietic precursors and maintenance of stem cells in the hematopoietic system. Transgenic mice lacking the sFRP1 gene, display niche-dependent self-renewal defects in their HSC, including quiescence loss and self-renewal impairment [6]. In hematopoietic malignancies, epigenetic downregulation of sFRPs are believed to play a role in disease pathogenesis [70]. Methylation rate of sFRP1 was reported to be $51 \%$ in ALL [71] and $100 \%$ in CLL [72]. Other studies also reinforce these observations, reporting promoter methylation and down regulation of sFRP1, 2, 4 and 5 in primary CLL cells [8].

In myeloid leukemia's, Reins et al. reported promoter methylation and reduced expression levels of the sFRP1 gene 121 patients with AML, ALL and MDS (myelodysplastic syndrome) at different risk groups [73]. Aberrant methylation of sFRP genes varies in all Fab-AML subgroups. Epigenetic silencing of sFRP 1, 3, 4 leading to Wnt pathway activation in AML [8]; where the highest incidence was observed in the M4 and M1 FAB subgroups [74]. sFRP 1, 2, 4 and 5 promoter regions were shown to be methylated in different AML cell lines and hypermethylation of at least one SFRP gene was reported to occurred in over $50 \%$ of AML patients. Guo et al. reported that AML patients in which the sFRP1 promoter region was hypermethylated, had high incidence of N/K-Ras mutations. They argued that hypermethylation of sFRPs should be considered as an independent prognostic biomarker among both cytogenetically normal AML patients and non-M3 AML patients [7]. Uhm et al. determined that sFRP1 gene promoter methylation in AML patients was significantly higher when compared to CML patients. They reported methylation of the SFRP1 promoter region in 15 of the 23 AML patients (65.2\%), compared to only 2 of the $21 \mathrm{CML}$ patients (9.5\%) studied [75].

We have previously reported that albeit the observed infrequency in chronic phase CML, when present sFRP1 promoter methylation correlated with primary cytogenetic resistance to imatinib mesylate [76]. We've also shown that the sFRP1 gene is hyper methylated in CML cell lines. Forced SFRP1 expression in CML cell lines will sensitize cells to tyrosine kinase inhibitors. When SFRP1 and WIF1 are forced to re-expressed, they increase the apoptotic susceptibility of the cells in response to tyrosine kinase inhibitors [27].

\subsubsection{SOST}

Sclerostin is the SOST gene product. It is expressed in several tissues including bone, liver cartilage, kidney, heart, lung, pancreas and skeletal muscle. Its function has been mostly studied in bone tissue. Bone tissue undergoes constant remodeling orchestrated mainly by three cell 
types: osteoclasts (bone resorbing cells), osteoblasts (responsible for the bone synthesis and mineralization) and osteocytes. In bone, sclerostin is primarily secreted by osteocytes; but osteoblasts and osteoclasts also express SOST at low levels [77]. Sclerostin was originally defined as a non-canonical bone morphogenetic protein (BMP) antagonist [78]. It has been classified as a Wnt antagonist due to its inhibitory effect on Wnt signaling. Circulating sclerostin binds to the LRP5/6 proteins through the PE domain and inhibits the formation of the LRP5/6-frizzled receptor complex; leading to phosphorylation and subsequent degradation of $\beta$-catenin and Wnt pathway inhibition $[46,77,79]$. Suppression of the Wnt pathway by sclerostatin leads to an decrease in bone development [80]. Due to this effect a human monoclonal sclerostin antibody which inhibits the binding of sclerostin to LRP5/6 has been evaluated as part of a randomized, double-blind placebo controlled phase I trial; and has shown promising results with increases in bone mass density [81]. Similar antibodies have been developed and phase II trials are underway. Nevertheless enhanced Wnt signaling has been linked to different malignancies therefore the long-term safety results of these therapeutic interventions are awaited.

There are only a limited number of papers of any role SOST may have in cancer. Research in this area is focused on bone tumors and skeletal sarcomas $[82,83]$. In the context of hematological malignancies, sclerostin antibodies were reported to suppress tumor growth in MM patients [84]. There are no reports on the role of SOST in leukomogenesis.

\subsubsection{Wnt5A}

The Wnt5a ligand is also a natural antagonist of Wnt signaling [8]. It has a role in both canonical and non-canonical Wnt pathways. Wnt5a activates Wnt/PCP pathway signaling via activating small Rho-GTPases [85]. On the other hand, Wnt5a has the ability to inhibit Wnt3a dependent LRP6 phosphorylation and $\beta$-catenin accumulation in vitro by competing with Wnt3a to bind to the Frizzled 2 receptor [86]. Evidence indicates that Wnt5a exhibits dual effects on carcinogenesis depending on context and tissue specificity. While favorable outcomes have been reported in different tumors with high Wnt5a expression [87]; there are also reports showing that over-expression of Wnt5a leads to a more aggressive phenotype $[88,89]$. One explanation to this observed discrepancy may be the involvement of different isoforms [90]. Nevertheless Wnt5a is emerging as an important player in cancer progression, exerting both oncogenic and tumor suppressive effects [86].

There are fewer studies on the role of Wnt5A in leukemia; but as in solid tumors, both oncogenic and tumor suppressive effects are observed. One study reported epigenetic changes of Wnt antagonists in acute leukemia and has shown that in most cases the Wnt5a promoter is methylated [55]. Hatırnaz et al. showed that Wnt5a hyper methylation has a direct effect on mRNA expression levels and Wnt5a acts as a tumor suppressor in ALL patients [91]. It was also reported to act as tumor suppressor in AML patients where the epigenetic inhibition of Wnt5a leads to upregulation of Cyclin D1 expression and a poor prognosis [92]. Exogenous Wnt5a expression enhances proliferation and migration of CLL cells[93]. On the other hand in CLL, Wnt-5a functions as an important regulator of ROR1 activity. Also autocrine Wnt5a was shown to induce irregular motility and chemotaxis in primary CLL cells [94]. Additionally, one report has shown that over expressing Wnt5a increases the effects of imatinib in CML cells through the JNK/ $\beta$-catenin/Survivin and $\gamma$-catenin/ $\beta$-catenin/Survivin pathways [95].

\subsection{Therapeutic potential of Wnt antagonists in Leukemia}

Research has revealed Wnt pathways are central to the malignant phenotype of many tumors. Thus many therapeutical approaches and agents have been developed -some currently in clinical trails- that specifically target one or more of the many players [96]. Re-expressing epigenetically inhibited Wnt antagonists is also among therapeutical strategies to be considered. Non-specific epigenetic modulation by using histon deacetylase and DNA methylase inhibitors is currently part of cancer therapy, including hematological malignancies. These agents lead to the re-expression of Wnt antagonisits as well as other silenced tumor suppressors. Specific targeting of antagonists is more challenging; hence epigenetic modulation of a specific promotor/s is technically much more difficult. In this context replacement approaches rather than epigenetic modulation are favored. SFRP1 has been used in the form of a recombinant therapeutic protein attached to gold nanoparticles that allowed release of sFRP1 outside the cells, resulting in the inhibition of the malignant phenotype [97]. Advancements in nanomedicine, where nanocarriers are taking center stage may lead to the replacement of specific molecules -such as Wnt antogonists- in targeted tissues. Viral vectors have also been used to deliver Wnt antagonists. The intratumoral injection of an adenovirus vector carrying the Dkk-3 gene was reported to suppresses tumors in mesothelioma, prostate, breast and testicular cancers [98]. Recombinant Dkk1 protein has been used as a canonical Wnt signalling inhibitor, in-vitro settings [50]. In addition, an adenosine analogue was shown to induce Dkk1 expression through autocrine and paracrine pathways in leukemia and mesenchymal stromal/stem cells (MSCs). MSCs that secrete Dkk1 was shown to inhibit proliferation in leukemia and cancer cells [58]. A few studies have examined the utility of short peptides. SFRP1 or SFRP1-derived peptides were shown to delay xenograft tumor formation in nude mice. Likewise, the injection of full-length SFRP1 protein in xenografted mammary tumors were shown to reduce tumor growth. Full-length WNT5A both normal mammary cells and breast cancer cells invitro and daily injections of a WNT5A-derived peptide reduced the number of metastases in mice allografted with breast cancer cells [99]. SOST is a exception among Wnt antagonists in that its inhibition -not activationbenefits clinical outcomes in cancer. its exerts its inhibitory effect on Wnt signaling binding to LRP5/6 proteins and inhibiting the formation of the LRP5/6-frizzled receptor complex. Yet it is classified as a It has been classified as a bone morphogenetic protein (BMP) antagonist with roles in bone development [78]. Suppression of the Wnt pathway by sclerostatin leads to an decrease in bone development [80]. SOST antibodies prevented bone loss and reduced osteolytic bone lesions in multiple myeloma [100]. A human monoclonal sclerostin antibody which inhibits the binding of sclerostin to LRP5/6 is currently evaluated for clinical use in phase I and II trails [81].

On-going and future advances in gene therapy, microRNA delivery/ efficiency, CRISPR/Cas technologies are all promising approaches to utilize therapeutic strategies to modulate Wnt antagonists in clinical setting and their use in hematological malignancies.

\subsection{Conclusion}

Although we've come a long way in treating leukemia's, research efforts are being focused on developing newer and better regimens. The Wnt signaling pathway is a crucial regulator of hematopoiesis and its dysfunction has been implicated in the development of leukemia's. In recent years, research has shown that epigenetic silencing of Wnt antagonists contributes to the abnormal activation of canonical Wnt signaling. Wnt signaling pathway antagonists plays multiple roles in human hematopoiesis. However, their precise roles and the causal mechanisms in leukemia have not been clearly elucidated. Fully understanding the function of these epigenetically silenced regulators enriches our repertoire of possible targets for therapy. Activated Wntsignaling may be a key molecular event, which is able to circumvent classical therapeutical approaches. Wnt antagonists may carry the potential to be exploited in designing new agents and strategies for the different forms of leukemia; while Wnt signaling may be a target, in therapy resistance disease.

\section{Declarations of interests}

None. 


\section{Funding}

This research did not receive any specific grant from funding agencies in the public, commercial, or not-for-profit sectors.

\section{Contributions}

MP, CC, ZY and HOS designed the study, searched databases, performed the selection of studies analyzed, drafted and wrote manuscript. All authors reviewed and approved the final manuscript.

\section{Acknowledgements}

The authors were fully responsible for content and editorial decisions for this manuscript and have approved of the final draft for submission.

\section{References}

[1] C.H. Jamieson, L.E. Ailles, S.J. Dylla, M. Muijtjens, C. Jones, J.L. Zehnder, et al., Granulocyte-macrophage progenitors as candidate leukemic stem cells in blastcrisis CML, New Engl. J. Med. 351 (7) (2004) 657-667.

[2] Z.-J. Kang, Y.-F. Liu, L.-Z. Xu, Z.-J. Long, D. Huang, Y. Yang, et al., The Philadelphia chromosome in leukemogenesis, Chin. J. Cancer 35 (1) (2016) 48.

[3] S. Salesse, C. Verfaillie, BCR/ABL: from molecular mechanisms of leukemia induction to treatment of chronic myelogenous leukemia, Oncogene 21 (56) (2002) 8547-8559.

[4] C.H. Jamieson, Chronic myeloid leukemia stem cells, ASH Educ. Program Book 2008 (1) (2008) 436-442.

[5] N. Khan, L. Bendall, Role of WNT signaling in normal and malignant hematopoiesis, Histol. Histopathol. 21 (7) (2006) 761-774.

[6] P.F.S. Etet, L. Vecchio, P.B. Kamga, E.N. Nukenine, M. Krampera, A.H.N. Kamdje, Normal hematopoiesis and hematologic malignancies: role of canonical Wnt signaling pathway and stromal microenvironment, Biochim. Biophys. Acta (BBA)-Rev. Cancer 1835 (1) (2013) 1-10.

[7] H. Guo, T-j. Zhang, X-m. Wen, J-d. Zhou, J-c. Ma, C. An, et al., hypermethylation of secreted frizzled-related proteins predicts poor prognosis in non-M3 acute myeloid leukemia, OncoTargets Ther. 10 (2017) 3635.

[8] F.J. Staal, F. Famili, L. Garcia Perez, K. Pike-Overzet, Aberrant wnt signaling in leukemia, Cancers 8 (9) (2016) 78.

[9] C.Y. Logan, R. Nusse, The Wnt signaling pathway in development and disease, Annu. Rev. Cell Dev. Biol. 20 (2004) 781-810.

[10] C.-S. Rhee, M. Sen, D. Lu, C. Wu, L. Leoni, J. Rubin, et al., Wnt and frizzled receptors as potential targets for immunotherapy in head and neck squamous cell carcinomas, Oncogene 21 (43) (2002) 6598.

[11] M. Kahn, Can we safely target the WNT pathway? Nat. Rev. Drug Discov. 13 (7) (2014) 513.

[12] J.L. Stamos, W.I. Weis, The $\beta$-catenin destruction complex, Cold Spring Harbor Perspect. Biol. 5 (1) (2013) a007898.

[13] C. Gao, G. Xiao, J. Hu, Regulation of Wnt/ $\beta$-catenin signaling by posttranslational modifications, Cell Biosci. 4 (1) (2014) 13.

[14] L.-J. Liu, S.-X. Xie, Y.-T. Chen, J.-L. Xue, C.-J. Zhang, F. Zhu, Aberrant regulation of Wnt signaling in hepatocellular carcinoma, World J. Gastroenterol. 22 (33) (2016) 7486.

[15] C.J. Cain, J.O. Manilay, Hematopoietic stem cell fate decisions are regulated by Wnt antagonists: comparisons and current controversies, Exp. Hematol. 41 (1) (2013) $3-16$.

[16] The role of extracellular modulators of canonical Wnt signaling in bone metabolism and diseases, in: E. Boudin, I. Fijalkowski, E. Piters, W. Van Hul (Eds.), Seminars in Arthritis and Rheumatism, Elsevier, 2013.

[17] M. Katoh, WNT/PCP signaling pathway and human cancer (review), Oncol. Rep. 14 (6) (2005) 1583.

[18] M.T. Veeman, J.D. Axelrod, R.T. Moon, A second canon: functions and mechanisms of $\beta$-catenin-independent Wnt signaling, Dev. Cell 5 (3) (2003) 367-377.

[19] H.W. Park, Y.C. Kim, B. Yu, T. Moroishi, J.-S. Mo, S.W. Plouffe, et al., Alternative wnt signaling activates YAP/TAZ, Cell 162 (4) (2015) 780-794.

[20] L. Azzolin, T. Panciera, S. Soligo, E. Enzo, S. Bicciato, S. Dupont, et al., YAP/TAZ incorporation in the $\beta$-catenin destruction complex orchestrates the Wnt response, Cell 158 (1) (2014) 157-170.

[21] H. Clevers, R. Nusse, Wnt/ß-catenin signaling and disease, Cell 149 (6) (2012) $1192-1205$.

[22] W. de Lau, N. Barker, T.Y. Low, B.-K. Koo, V.S. Li, H. Teunissen, et al., Lgr5 homologues associate with Wnt receptors and mediate R-spondin signalling, Nature 476 (7360) (2011) 293-297.

[23] R. Nusse, H. Clevers, Wnt/ $\beta$-catenin signaling, disease, and emerging therapeutic modalities, Cell 169 (6) (2017) 985-999.

[24] T. Reya, A.W. Duncan, L. Ailles, J. Domen, A role for Wnt signalling in self-renewal of haematopoietic stem cells, Nature 423 (6938) (2003) 409.

[25] G. Xueling, X. Wang, Role of Wnt canonical pathway in hematological malignancies, J. Hematol. Oncol. 3 (1) (2010) 33.
[26] E.A. Griffiths, S.D. Gore, C. Hooker, M.A. McDevitt, J.E. Karp, B.D. Smith, et al., Acute myeloid leukemia is characterized by Wnt pathway inhibitor promoter hypermethylation, Leukemia Lymphoma 51 (9) (2010) 1711-1719.

[27] M. Pehlivan, C. Caliskan, Z. Yuce, H.O. Sercan, Forced expression of Wnt antagonists SFRP1 and WIF1 sensitizes chronic myeloid leukemia cells to tyrosine kinase inhibitors, Tumor Biol. 39 (5) (2017) 1010428317701654.

[28] W. Lento, K. Congdon, C. Voermans, M. Kritzik, T. Reya, Wnt signaling in normal and malignant hematopoiesis, Cold Spring Harbor Perspect. Biol. 5 (2) (2013) a008011.

[29] F.M. Rattis, C. Voermans, T. Reya, Wnt signaling in the stem cell niche, Curr. Opin. Hematol. 11 (2) (2004) 88-94.

[30] T.C. Luis, M. Ichii, M.H. Brugman, P. Kincade, F.J. Staal, Wnt signaling strength regulates normal hematopoiesis and its deregulation is involved in leukemia development, Leukemia 26 (3) (2012) 414-421.

[31] R.B. Undi, U. Gutti, I. Sahu, S. Sarvothaman, S.R. Pasupuleti, R. Kandi, et al., Wnt signaling: role in regulation of haematopoiesis, Indian J. Hematol. Blood Transfusion 32 (2) (2016) 123-134.

[32] M.C. Florian, K.J. Nattamai, K. Dörr, G. Marka, B. Überle, V. Vas, et al., A canonical to non-canonical Wnt signalling switch in haematopoietic stem-cell ageing, Nature 503 (7476) (2013) 392

[33] R. Sugimura, X.C. He, A. Venkatraman, F. Arai, A. Box, C. Semerad, et al., Noncanonical Wnt signaling maintains hematopoietic stem cells in the niche, Cell 150 (2) (2012) 351-365.

[34] T. Sadras, R.J. D'Andrea, D.L. White, The role of wnt/ $\beta$-catenin signaling in normal and malignant hematopoiesis, hemostasis, J. Blood Res. Hematol. Dis. (2017) 2016.

[35] E. Ashihara, T. Takada, T. Maekawa, Targeting the canonical Wnt/ $\beta$-catenin pathway in hematological malignancies, Cancer Sci. 106 (6) (2015) 665-671.

[36] Y. Wang, A.V. Krivtsov, A.U. Sinha, T.E. North, W. Goessling, Z. Feng, et al., The Wnt/ $\beta$-catenin pathway is required for the development of leukemia stem cells in AML, Science 327 (5973) (2010) 1650-1653.

[37] F.H. Heidel, B.G. Mar, S.A. Armstrong, Self-renewal related signaling in myeloid leukemia stem cells, Int. J. Hematol. 94 (2) (2011) 109.

[38] Y. Ying, Q. Tao, Epigenetic disruption of the WNT/ß-catenin signaling pathway in human cancers, Epigenetics 4 (5) (2009) 307-312.

[39] H. Liang, Q. Chen, A.H. Coles, S.J. Anderson, G. Pihan, A. Bradley, et al., Wnt5a inhibits B cell proliferation and functions as a tumor suppressor in hematopoietic tissue, Cancer Cell 4 (5) (2003) 349-360.

[40] V. Martin, X. Agirre, A. Jiménez-Velasco, E.S. José-Eneriz, L. Cordeu, L. Gárate, et al., Methylation status of Wnt signaling pathway genes affects the clinical outcome of Philadelphia-positive acute lymphoblastic leukemia, Cancer Sci. 99 (9) (2008) 1865-1868.

[41] M.A. Gregory, T.L. Phang, P. Neviani, F. Alvarez-Calderon, C.A. Eide, T. O'Hare, et al., Wnt/Ca $2+$ /NFAT signaling maintains survival of $\mathrm{Ph}+$ leukemia cells upon inhibition of Bcr-Abl, Cancer Cell 18 (1) (2010) 74-87.

[42] P. Janovska, J. Verner, J. Kohoutek, L. Bryjova, M. Gregorova, M. Dzimkova, et al., Casein kinase 1 is a therapeutic target in chronic lymphocytic leukemia, Blood 131 (11) (2018) 1206-1218.

[43] S. Baskar, K.Y. Kwong, T. Hofer, J.M. Levy, M.G. Kennedy, E. Lee, et al., Unique cell surface expression of receptor tyrosine kinase ROR1 in human B-cell chronic lymphocytic leukemia, Clin. Cancer Res. 14 (2) (2008) 396-404.

[44] A.H. DaneshManesh, E. Mikaelsson, M. Jeddi-Tehrani, A.A. Bayat, R. Ghods, M. Ostadkarampour, et al., Ror1, a cell surface receptor tyrosine kinase is expressed in chronic lymphocytic leukemia and may serve as a putative target for therapy, Int. J. Cancer 123 (5) (2008) 1190-1195.

[45] T. Fukuda, L. Chen, T. Endo, L. Tang, D. Lu, J.E Castro, et al, Antisera induced by infusions of autologous Ad-CD154-leukemia B cells identify ROR1 as an oncofetal antigen and receptor for Wnt5a, Proc. Natl. Acad. Sci. 105 (8) (2008) 3047-3052.

[46] C.-M. Cruciat, C. Niehrs, Secreted and transmembrane wnt inhibitors and activators, Cold Spring Harbor Perspect. Biol. 5 (3) (2013) a015081.

[47] C. Niehrs, Function and biological roles of the Dickkopf family of Wnt modulators, Oncogene 25 (57) (2006) 7469-7481.

[48] Y. Zhang, H. Li, R. Cao, L. Sun, Y. Wang, S. Fan, et al., Suppression of miR-708 inhibits the Wnt/ $\beta$-catenin signaling pathway by activating DKK3 in adult B-all, Oncotarget 8 (38) (2017) 64114.

[49] A. Niida, T. Hiroko, M. Kasai, Y. Furukawa, Y. Nakamura, Y. Suzuki, et al., DKK1, a negative regulator of Wnt signaling, is a target of the $\beta$-catenin/TCF pathway, Oncogene 23 (52) (2004) 8520-8526.

[50] S. Pozzi, M. Fulciniti, H. Yan, S. Vallet, H. Eda, K. Patel, et al., In vivo and in vitro effects of a novel anti-Dkk1 neutralizing antibody in multiple myeloma, Bone 53 (2) (2013) 487-496.

[51] H. Hirata, Y. Hinoda, K. Nakajima, K. Kawamoto, N. Kikuno, K. Ueno, et al., Wnt antagonist DKK1 acts as a tumor suppressor gene that induces apoptosis and inhibits proliferation in human renal cell carcinoma, Int. J. Cancer 128 (8) (2011) 1793-1803.

[52] M. Zebisch, V.A. Jackson, Y. Zhao, E.Y. Jones, Structure of the dual-mode wnt regulator kremen1 and insight into ternary complex formation with LRP6 and dickkopf, Structure 24 (9) (2016) 1599-1605.

[53] T.H. Kim, J.S. Chang, K.-S. Park, J. Park, N. Kim, J.I. Lee, et al., Effects of exercise training on circulating levels of Dickkpof- 1 and secreted frizzled-related protein-1 in breast cancer survivors: a pilot single-blind randomized controlled trial, PLoS One 12 (2) (2017) e0171771.

[54] B. Mao, C. Niehrs, Kremen2 modulates Dickkopf2 activity during Wnt/LRP6 signaling, Gene 302 (1) (2003) 179-183.

[55] H.-R. Zhou, H.-Y. Fu, D.-S. Wu, Y.-Y. Zhang, S.-H. Huang, C.-J. Chen, et al., Relationship between epigenetic changes in Wnt antagonists and acute leukemia, Oncol. Rep. 37 (5) (2017) 2663-2671. 
[56] N.C. Munshi, K.C. Anderson, Advances in Biology and Therapy of Multiple Myeloma: Volume 1: Basic Science, Springer Science \& Business Media, 2012.

[57] E. Tian, F. Zhan, R. Walker, E. Rasmussen, Y. Ma, B. Barlogie, et al., The role of the Wnt-signaling antagonist DKK1 in the development of osteolytic lesions in multiple myeloma, New Engl. J. Med. 349 (26) (2003) 2483-2494.

[58] S.-M. Liang, Y.-J. Lu, B.-S. Ko, Y.-J. Jan, S.-K. Shyue, S.-F. Yet, et al., Cordycepin disrupts leukemia association with mesenchymal stromal cells and eliminates leukemia stem cell activity, Sci. Rep. (2017) 7.

[59] A. Filipovich, R.K. Gandhirajan, I. Gehrke, S.J. Poll-Wolbeck, K.A. Kreuzer, Evidence for non-functional Dickkopf-1 (DKK-1) signaling in chronic lymphocytic leukemia (CLL), Eur. J. Haematol. 85 (4) (2010) 309-313.

[60] T. Malinauskas, A.R. Aricescu, W. Lu, C. Siebold, E.Y. Jones, Modular mechanism of Wnt signaling inhibition by Wnt inhibitory factor 1, Nat. Struct. Mol. Biol. 18 (8) (2011) 886-893.

[61] J. Veeck, P.J. Wild, T. Fuchs, P.J. Schüffler, A. Hartmann, R. Knüchel, et al., Prognostic relevance of Wnt-inhibitory factor-1 (WIF1) and Dickkopf-3 (DKK3) promoter methylation in human breast cancer, BMC Cancer 9 (1) (2009) 217.

[62] Y. Kawano, R. Kypta, Secreted antagonists of the Wnt signalling pathway, J. Cell Sci. 116 (13) (2003) 2627-2634.

[63] K. Kawakami, H. Hirata, S. Yamamura, N. Kikuno, S. Saini, S. Majid, et al., Functional significance of Wnt inhibitory factor-1 gene in kidney cancer, Cancer Res. 69 (22) (2009) 8603-8610.

[64] C. Chim, T. Fung, K. Wong, J. Lau, R. Liang, Infrequent Wnt inhibitory factor-1 (Wif-1) methylation in chronic lymphocytic leukemia, Leukemia Res. 30 (9) (2006) $1135-1139$.

[65] A. Ghasemi, A. Ghotaslou, M. Mohammadi, S. Abbasian, K. Ghaffari, Methylation of the Wnt signaling antagonist, Wnt inhibitory factor 1 and dickkopf-1 genes in acute myeloid leukemia at the time of diagnosis, Zahedan J. Res. Med. Sci. 18 (1) (2016).

[66] S. Warrier, R. Marimuthu, S. Sekhar, G. Bhuvanalakshmi, F. Arfuso, A.K. Das, et al., sFRP-mediated Wnt sequestration as a potential therapeutic target for Alzheimer's disease, Int. J. Biochem. Cell Biol. 75 (2016) 104-111.

[67] S.E. Jones, C. Jomary, Secreted Frizzled-related proteins: searching for relationships and patterns, Bioessays 24 (9) (2002) 811-820.

[68] Y. Wang, Y.-P. Li, C. Paulson, J.-Z. Shao, X. Zhang, M. Wu, et al., Wnt and the Wnt signaling pathway in bone development and disease, Front. Biosci. (Landmark ed.) 19 (2014) 379.

[69] M. Nojima, H. Suzuki, M. Toyota, Y. Watanabe, R. Maruyama, S. Sasaki, et al, Frequent epigenetic inactivation of SFRP genes and constitutive activation of Wnt signaling in gastric cancer, Oncogene 26 (32) (2007) 4699-4713.

[70] K. Bennemann, O. Galm, S. Wilop, C. Schubert, T.H. Brümmendorf, E. Jost, Epigenetic dysregulation of secreted frizzled-related proteins in myeloproliferative neoplasms complements the JAK2V617F-mutation, Clin. Epigenet. 4 (1) (2012) 12.

[71] J. Roman-Gomez, A. Jimenez-Velasco, X. Agirre, F. Prosper, A. Heiniger, A. Torres, Lack of $\mathrm{CpG}$ island methylator phenotype defines a clinical subtype of T-cell acute lymphoblastic leukemia associated with good prognosis, J. Clin. Oncol. 23 (28) (2005) 7043-7049.

[72] T.-H. Liu, A. Raval, S.-S. Chen, J.J. Matkovic, J.C. Byrd, C. Plass, CpG island methylation and expression of the secreted frizzled-related protein gene family in chronic lymphocytic leukemia, Cancer Res. 66 (2) (2006) 653-658.

[73] J. Reins, M. Mossner, M. Neumann, U. Platzbecker, C. Schumann, E. Thiel, et al. Transcriptional down-regulation of the Wnt antagonist SFRP1 in haematopoietic cells of patients with different risk types of MDS, Leuk. Res. 34 (12) (2010) 1610-1616.

[74] A. Ghasemi, S. Rostami, B. Chahardouli, N.A. Ghandforosh, A. Ghotaslou, F. Nadali, Study of SFRP1 and SFRP2 methylation status in patients with de novo Acute Myeloblastic Leukemia, Int. J. Hematol.-Oncol. Stem Cell Res. 9 (1) (2015) 15.

[75] K.-O. Uhm, E.S. Lee, Y.M. Lee, J.S. Park, S.J. Kim, B.S. Kim, et al., Differential methylation pattern of ID4, SFRP1, and SHP1 between acute myeloid leukemia and chronic myeloid leukemia, J. Korean Med. Sci. 24 (3) (2009) 493-497.

[76] M. Pehlivan, Z. Sercan, Sercan HO: sFRP1 promoter methylation is associated with persistent Philadelphia chromosome in chronic myeloid leukemia, Leuk. Res. 33 (8) (2009) 1062-1067.

[77] A. Sebastian, G.G. Loots, Genetics of Sost/SOST in sclerosteosis and van buchem disease animal models, Metabolism 80 (2017) 38-47.

[78] D.G. Winkler, M.K. Sutherland, J.C. Geoghegan, C. Yu, T. Hayes, J.E. Skonier, et al.,
Osteocyte control of bone formation via sclerostin, a novel BMP antagonist, EMBO J. 22 (23) (2003) 6267-6276.

[79] M.K. Mohammed, C. Shao, J. Wang, Q. Wei, X. Wang, Z. Collier, et al., Wnt/ßcatenin signaling plays an ever-expanding role in stem cell self-renewal, tumorigenesis and cancer chemoresistance, Genes Dis. 3 (1) (2016) 11-40.

[80] M. Semënov, K. Tamai, X. He, SOST is a ligand for LRP5/LRP6 and a Wnt signaling inhibitor, J. Biol. Chem. 280 (29) (2005) 26770-26775.

[81] S. Ishtiaq, I. Fogelman, G. Hampson, Treatment of post-menopausal osteoporosis: beyond bisphosphonates, J. Endocrinol. Invest. 38 (1) (2015) 13-29.

[82] Y. Inagaki, E.S. Hookway, T.G. Kashima, M. Munemoto, Y. Tanaka, A.B. Hassan, et al., Sclerostin expression in bone tumours and tumour-like lesions, Histopathology 69 (3) (2016) 470-478.

[83] J. Shen, C.A. Meyers, S. Shrestha, A. Singh, G. LaChaud, V. Nguyen, et al., Sclerostin expression in skeletal sarcomas, Hum. Pathol. 58 (2016) 24-34.

[84] M.R. Reagan, M. McDonald, R. Terry, J. Pettitt, L. Le, S. Mohanty, et al., Antisclerostin treatment prevents multiple myeloma induced bone loss and reduces tumor burden, Blood 126 (2015) 119.

[85] X. Deng, Z. Tu, M. Xiong, K. Tembo, L. Zhou, P. Liu, et al., Wnt5a and CCL25 promote adult T-cell acute lymphoblastic leukemia cell migration, invasion and metastasis, Oncotarget 8 (24) (2017) 39033.

[86] Y. Zhou, T.J. Kipps, S. Zhang, Wnt5a signaling in normal and cancer stem cells, Stem Cells Int. (2017) 2017.

[87] G. Canesin, S. Evans-Axelsson, R. Hellsten, A. Krzyzanowska, C.P. Prasad, A. Bjartell, et al., Treatment with the WNT5A-mimicking peptide Foxy-5 effectively reduces the metastatic spread of WNT5A-low prostate cancer cells in an orthotopic mouse model, PLoS One 12 (9) (2017) e0184418.

[88] B. Wang, Z. Tang, H. Gong, L. Zhu, X. Liu, Wnt5a promotes epithelial-to-mesenchymal transition and metastasis in non-small cell lung cancer, Biosci. Rep. 37 (6) (2017) BSR20171092.

[89] Y. Wang, C. Xu, Y. Wang, X. Zhang, MicroRNA-365 inhibits ovarian cancer progression by targeting Wnt5a, Am. J. Cancer. Res. 7 (5) (2017) 1096.

[90] T.-C. Huang, P.-T. Lee, M.-H. Wu, C.-C. Huang, C.-Y. Ko, Y.-C. Lee, et al., Distinct roles and differential expression levels of Wnt5a mRNA isoforms in colorectal cancer cells, PLoS One 12 (8) (2017) e0181034.

[91] Ng ÖH, S. Fırtına, Can İ, Z. Karakaş, L. Ağaoğlu, Doğru Ö, et al., A possible role for WNT5A hypermethylation in pediatric acute lymphoblastic leukemia, Turk. J. Hematol. 32 (2) (2015) 127.

[92] V. Martín, A. Valencia, X. Agirre, J. Cervera, E.S. Jose-Eneriz, A. Vilas-Zornoza, et al., Epigenetic regulation of the non-canonical Wnt pathway in acute myeloid leukemia, Cancer Sci. 101 (2) (2010) 425-432.

[93] J. Yu, L. Chen, B. Cui, G.F. Widhopf, Z. Shen, R. Wu, et al., Wnt5a induces ROR1/ ROR2 heterooligomerization to enhance leukemia chemotaxis and proliferation, J. Clin. Invest. 126 (2) (2016) 585-598.

[94] P. Janovska, L. Poppova, K. Plevova, H. Plesingerova, M. Behal, M. Kaucka, et al, Autocrine signaling by Wnt-5a deregulates chemotaxis of leukemic cells and predicts clinical outcome in chronic lymphocytic leukemia, Clin. Cancer Res. 0154 (2015) 2015 (clincanres).

[95] C.-C. Niu, C. Zhao, X.-L. Zhang, J. Pan, W.-R. Wu, Z.-Q. Li, et al., Wnt5a enhances the response of CML cells to Imatinib Mesylate through JNK activation and $\gamma$-catenin inhibition, Leuk. Res. 37 (11) (2013) 1532-1537.

[96] X. Zhang, J. Hao, Development of anticancer agents targeting the Wnt/ $\beta$-catenin signaling, Am. J. Cancer. Res. 5 (8) (2015) 2344.

[97] A. Ghoshal, U. Goswami, A.K. Sahoo, A. Chattopadhyay, S.S. Ghosh, Targeting Wnt canonical signaling by recombinant SFRP1 bound luminescent au-nanocluster embedded nanoparticles in cancer theranostics, ACS Biomater. Sci. Eng. 1 (12) (2015) $1256-1266$.

[98] M. Watanabe, Y. Nasu, H. Kumon, Adenovirus-mediated REIC/Dkk-3 gene therapy: development of an autologous cancer vaccination therapy, Oncol. Lett. 7 (3) (2014) 595-601.

[99] J.N. Anastas, R.T. Moon, WNT signalling pathways as therapeutic targets in cancer, Nat. Rev. Cancer 13 (1) (2013) 11.

[100] M.M. McDonald, M.R. Reagan, S.E. Youlten, S.T. Mohanty, A. Seckinger, R.L. Terry, et al., Inhibiting the osteocyte-specific protein sclerostin increases bone mass and fracture resistance in multiple myeloma, Blood 129 (26) (2017) 3452-3464. 